\title{
Effects of Flame Retardants Additives on the Properties of Low- Density Polyethylene
}

\author{
${ }^{*}$ Raed Ma'ali, ${ }^{1}$ Omar Surkhi, ${ }^{2}$ Shadi Sawalha, ${ }^{1}$ Sadiah Jibreeni, ${ }^{1}$ Suhair Fanoun, ${ }^{1}$ Anwar \\ Al-Khatib \\ ${ }^{l}$ Materials Engineering Department, Al-Quds Universiy, Abu Dies, Palestine. \\ ${ }^{2}$ Chemical Engineering Department, An-Najah National University, Nablus, Palestine. \\ *rmali@staff.alquds.edu, Telephone: +97022899807, Fax: +97022644210
}

\begin{abstract}
Low-density polyethylene (LDPE) has many unique properties such as lightweight and high chemical resistance. Unfortunately, it burns rapidly when it is exposed to a flame which limits its applications especially when flame resistance is to be considered. Different percentages of magnesium hydroxide and decabromide diphenyl ether (3.0, 5.0, 7.0, and $9.0 \mathrm{wt} . \%)$ were mixed with LDPE using a two-roll mill machine at $160^{\circ} \mathrm{C}$ for 2 minutes. Then, the tensile and flame retardancy tests samples were prepared by an injection molding process using an industrial plastic machine at $160^{\circ} \mathrm{C}$. Flammability, rheological, tensile and thermal properties of the produced samples were tested using a flammability test apparatus, a melt flow index machine, a universal testing machine, and a differential scanning calorimeter, respectively. It was observed that the flame resistance of LDPE was improved with the addition of both flame retardants up to $7.0 \mathrm{wt} . \%$, then it was reduced when $9.0 \mathrm{wt} . \%$ of flame retardants were used. This may be attributed to the poor mixing due to the increase in the polymer melt viscosity as observed from the melt flow index results. An increase in elastic modulus and a reduction in ductility of LDPE were observed with the increasing of flame-retardant contents while the ultimate tensile strength of LDPE was increased from 5.7 to 7.6 and $7.5 \mathrm{MPa}$ when $9.0 \mathrm{wt} . \%$ and $7.0 \mathrm{wt} . \%$ decabromide diphenyl ether and magnesium hydroxide were added. This is due to the fact that the additives act as a load carried and/or their effects on the degree of crystallinity of LDPE.
\end{abstract}

Indexed Terms: Low-density polyethylene, Flame-retardants, Melt flow index, Tensile properties, Thermal properties.

\section{INTRODUCTION}

Polymers are used in many applications due to the numerous properties that make them superior to other materials in many applications. Polyethylene (PE) is widely used due to its mechanical durability, good chemical resistance, low density, low toxicity, good electric insulation, low cost and ease of processing. But like the majority of other organic compounds, polyethylene will burn rapidly in an oxygen-full atmosphere. The flammability of polymers is a serious issue and severely limits their applications. Therefore, flame-retardants, such as halogenated additives, are used to improve the flame retardancy of polymers.

Several types of additives such as nanofiller, organo-modified clay montmorillonite with aluminum hydroxide, organically modified phyllosilicates, zinc borate, ammonium phosphate, iron powder, silicon, and phosphorous and nitrogen compounds were used to reduce flame retardancy of polymeric materials such as polyethylene, polypropylene and epoxy resin [1-7]. The of flame retardancy of the polymers was found to be affected by both the flame-retardant type and its content.

Extrusion technology was adopted to synthesize a flame retardant based on the esterification of melamine phosphate and pentaerythritol. An extruder was used to mix pentaerythritol and PE, and then copper chelates (salicylaldehyde and salicylaldoxime) were introduced into the ammonium polyphosphate to yield an intumescent polyethylene at a concentration of $0.2 \mathrm{wt} . \%$. Using a two-roll mill machine, the polymeric sheets were prepared by using a hot press at $150-160^{\circ} \mathrm{C}$ under $10 \mathrm{MPa}$ for 
5 minutes. The heat release rate, mass loss rate, fire growth rate and carbon monoxide emissions were reduced [8].

R.U. Arinze et al studied the effect of aluminum hydroxide, as a flame-retardant, on the flame characteristics of high-density polyethylene (HDPE) and polypropylene (PP). Aluminum hydroxide was added with different percentages $(0.5,1.0,1.5,2.0$ and $2.5 \mathrm{wt} . \%)$. It was found that the incorporation of the flame-retardant to HDPE and PP increased the ignition time as the concentration of the flame-retardant increases. This is due to the decomposition of the aluminum hydroxide during polymer combustion, which produces water vapor that can absorb heat thus providing a cooling effect [9].

The effects of sepiolite as a synergistic agent on the flame retardancy of polypropylene were studied. The flame-retardant was mixed with polypropylene by using a high-speed mixer, and then samples were extruded by a co-rotating twin-screw extruder and cut into pellets using a granulator. The resulting sample bars for testing were prepared by injection molding. The results showed that the addition of sepiolite to polypropylene has synergistic flame-retardant effects with the polymeric matrix [10].

This current study was carried out with the collaboration of Omega Plast. for Electrical and Communication Insulating Pipes, which produces LDPE pipes for electrical insulation. Due to the importance of the flame retardancy of these LDPE pipes, magnesium hydroxide or decabromide diphenyl ether is added to improve the flame retardancy of LDPE. The main objective of this work is to investigate the effects of magnesium hydroxide and decabromide diphenyl ether contents on the flame retardancy, rheological, tensile and thermal properties of LDPE.

\section{MATERIALS AND METHODS}

\subsection{Materials}

Low-density polyethylene, magnesium hydroxide and decabromide diphenyl ether were supplied by Omega Plast. For Electrical and Communication Insulating Pipes.

\subsection{Samples Preparation}

A home-made two-roll mill machine was used to mix the flame-retardants with LDPE. It consists of two stainless steel rolls that rotate in opposite directions at a fixed speed of rotation. It is equipped with electrical heaters in order to melt the polymeric materials. The processing variables such as the processing temperature, the speed of rotation, the distance between the rolls and the mixing time were controlled. LDPE pellets and different percentages of flame-retardants (3.0, 5.0, 7.0 and $9.0 \mathrm{wt} . \%)$ were used to prepare the samples. Firstly, LDPE pellets were melted using the two-roll mill machine at $140^{\circ} \mathrm{C}$ and low speed for 2 minutes, then the additives were gradually added and then mixed with the melt for another 2 minutes. After that, the mixture was obtained and cooled at room temperature. The dog bone and flame test samples were prepared by using Industrial Plastic Center (T013-P10) and injection molding model (T9013-P2). The temperatures of barrel and nozzle were fixed at $160^{\circ} \mathrm{C}, 165^{\circ} \mathrm{C}$, respectively. Each sample was heated in the industrial plastic center for 10 minutes, and then the polymer melt was injected in the suitable mold by using a pressure of 6 bars.

\subsection{Samples Characterizations}

The produced samples were tested to investigate the effect of flame-retardants on the flammability, rheological, thermal and tensile properties of LDPE.

\subsubsection{Flammability Test}

Flammability is the ability of a substance to burn or ignite; a fire test can be conducted to determine the degree of flammability. The flammability test was done according to the FTT UL 94 tests for flammability of plastic materials.

The test was carried out using the following steps: 
1. A test specimen bar of $13 \mathrm{~mm}$ by $125 \mathrm{~mm}$ by varying thickness was ignited while suspended at 10 $\mathrm{mm}$ above a calibrate methane burner, the flammability test apparatus is shown in Figure 1.

2. The flame was applied to a total of five test specimens twice for 10 seconds.

3. The burning time was recorded after each flame application for each test bar.

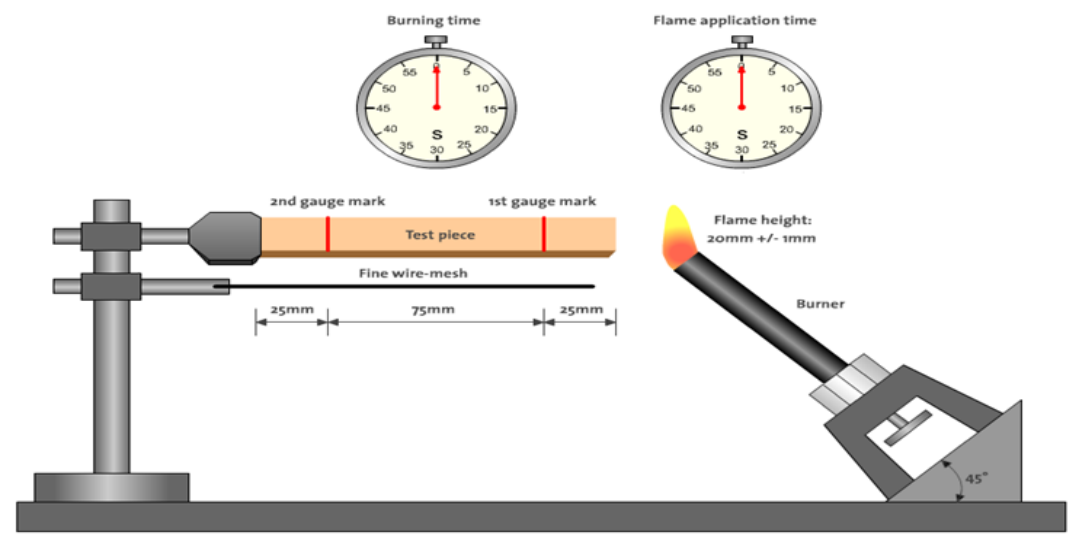

Figure 1: The flammability test apparatus.

\subsubsection{Melt Flow Index}

The melt flow index (MFI) is a measure of the ease of flow of the polymer melt. It is defined as the mass of polymer melt, in grams, flowing in ten minutes through a capillary of a specific diameter and length by a pressure applied by using prescribed gravimetric weights at a specific temperature. A home-made melt flow index was used to measure the melt flow index of the prepared samples, it consists of a metallic capillary that has a length of $15.3 \mathrm{~cm}$ and a diameter of $9.75 \mathrm{~mm}$, while the die has a diameter of $2.7 \mathrm{~mm}$. Ten grams each sample was packed inside the melt flow index machine, then the sample was heated for 10 minutes at $150^{\circ} \mathrm{C}$, after that a standard mass of $2.16 \mathrm{~kg}$ was introduced onto the piston. The mass of the obtained polymer melt at 10 minutes was measured by using an electrical balance (CPA423S, No26211484). The test was carried out with regard to ASTM D1238.

\subsubsection{Thermal Properties}

Thermal properties of the produced samples were measured by using differential scanning calorimeter (DSC) model Pyrix-6, PerkinElmer Corporation, U.K. A mass of 5 to $8 \mathrm{mg}$ from each sample was placed in a sealed aluminum pan. The same temperature profile (from room temperature to $200^{\circ} \mathrm{C}$ ), heated at $10^{\circ} \mathrm{C} / \mathrm{min}$ was applied to all samples. DSC test was done with regard to ASTM D3418-15 (Standard Test Method for Transition Temperatures and Enthalpies of Fusion and Crystallization of Polymers by Differential Scanning Calorimetry). Melting temperature and heat of fusion of the samples were obtained from the maximum peak and area under the peak, respectively. The latter was essential to estimate the percentage of crystalline regions. The recrystallization temperature was obtained from the maximum peak during the cooling stage.

\subsubsection{Tensile Properties}

A tensile test was carried out by using the universal testing machine (Gunt Hamburg apparatus WP 310 machine) at a constant speed of $5 \mathrm{~mm} / \mathrm{min}$ at room temperature. For each sample, five specimens of 50 $\mathrm{mm}$ gauge length, $8.0 \mathrm{~mm}$ width and $2.0 \mathrm{~mm}$ thickness were tested. The test was carried out according to a standard test method for tensile properties of plastic ASTM D638-14. 


\section{RESULTS AND DISCUSSION}

\subsection{Flammability Test}

Pure LDPE samples were found to burn spontaneously after first 10 seconds flame application, which continues until the test specimen was completely burnt up to the sample holding clamp, this means that the LDPE is not self-extinguishing. The effects of flame-retardant type and its content on the burning time of the LDPE are shown in Table 1.

Table 1: The burning time for samples containing flame-retardants

\begin{tabular}{|c|c|c|c|}
\hline $\begin{array}{c}\text { Magnesium hydroxide } \\
\text { content (wt.\%) }\end{array}$ & Time (Second) & $\begin{array}{c}\text { Decabromide diphenyl } \\
\text { ether content (wt. } \%)\end{array}$ & Time (Second) \\
\hline 0.0 & $42.4 \pm 3.8$ & 0.0 & $42.4 \pm 3.8$ \\
\hline 3.0 & $40.2 \pm 3.0$ & 3.0 & $11.4 \pm 2.8$ \\
\hline 5.0 & $21.3 \pm 2.6$ & 5.0 & $9.5 \pm 2.1$ \\
\hline 7.0 & $15.8 \pm 3.3$ & 7.0 & $3.9 \pm 1.5$ \\
\hline 9.0 & $33.9 \pm 3.5$ & 9.0 & $7.9 \pm 1.3$ \\
\hline
\end{tabular}

It can be observed from Table 1 that the addition of both magnesium hydroxide and decabromide diphenyl ether to LDPE improves its flame retardancy, the optimum value for each flame-retardant was observed at $7 \mathrm{wt} . \%$, but the flame retardancy for the samples containing decabromide diphenyl ether is better than the samples containing magnesium hydroxide. Magnesium hydroxide improves the flame resistance of LDPE because it decomposes when it is heated to produce magnesium oxide and water vapor. The gaseous water phase envelops the flame, thereby excluding oxygen and diluting flammable gases. A heat insulating material may form on the surface of the polymer in contact with the flame, reducing the flow of potentially flammable decomposition products to the gas phase where combustion occurs. It was reported that the use of metallic hydroxides such as aluminum and magnesium hydroxides was found to improve the flame retardancy of high-density polyethylene and polypropylene [9-10]. Decabromide phenyl ether is a halogenated flame-retardant $\left(\mathrm{C}_{12} \mathrm{Br}_{10} \mathrm{O}\right)$ which vaporizes in a similar temperature range as that of the plastic resin and so the halogen decreases the concentration of high energy free radicals that are involved in the combustion process. Eliminating these free radicals reduces flame intensity, decreases the amount of heat transferred to the plastic, and consequently slows or eliminates the burning of the plastic. The use of flame-retardants containing halogens was reported, the effectiveness of flame-retardants was found to be affected by both the halogen type and its content [12].

\subsection{Melt Flow Index}

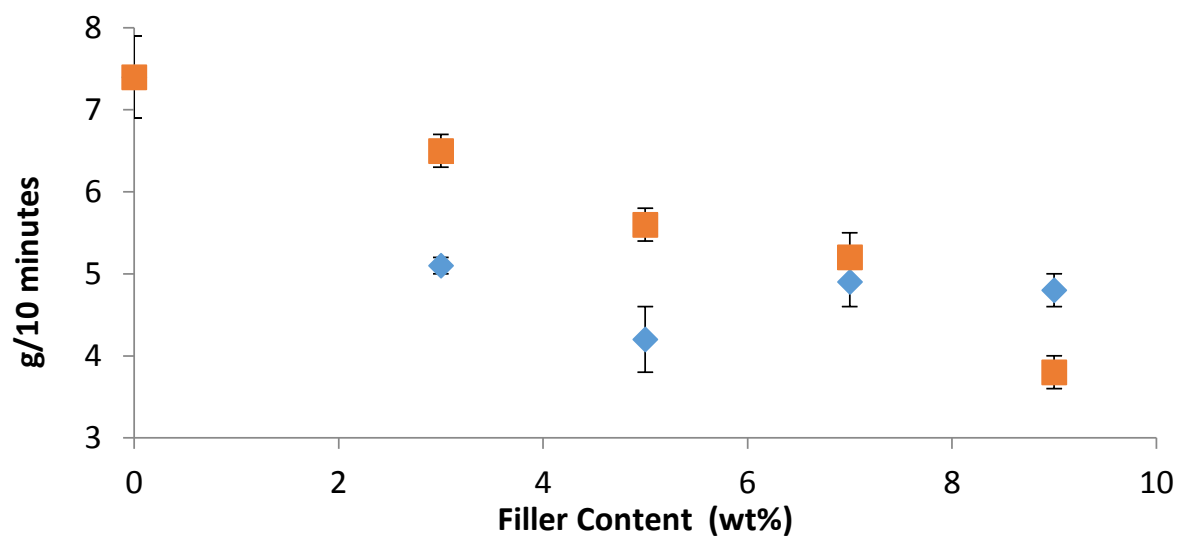

Figure 2: Effect of flame-retardants on MFI of LDPE composites, where and $\square$ represent magnesium hydroxide-containing samples and decabromide diphenyl ether samples, respectively. 
The melt flow index of LDPE containing flame retardants is shown in Figure 2, it can be seen that the melt flow index of LDPE decreased while increasing the amount of flame-retardants additives due to the increase in the polymer melt viscosity. The maximum reduction of viscosity was attended when 9.0 wt.\% flame-retardants additives were used, so the reduction in the flame retardancy of LDPE containing $9.0 \mathrm{wt} . \%$ flame-retardants additives may be related to the poor mixing of the additives with the polymer melt due to the increase in the polymer melt viscosity. The reduction of melt flow index of LDPE with the addition of ammonium phosphate as a flame retardant was reported [13].

\subsection{Thermal Properties}

Pure LDPE and samples containing the flame-retardants were scanned by a DSC from room temperature to $200{ }^{\circ} \mathrm{C}$, the heating curves of the samples are shown in Figures 3 and 4.

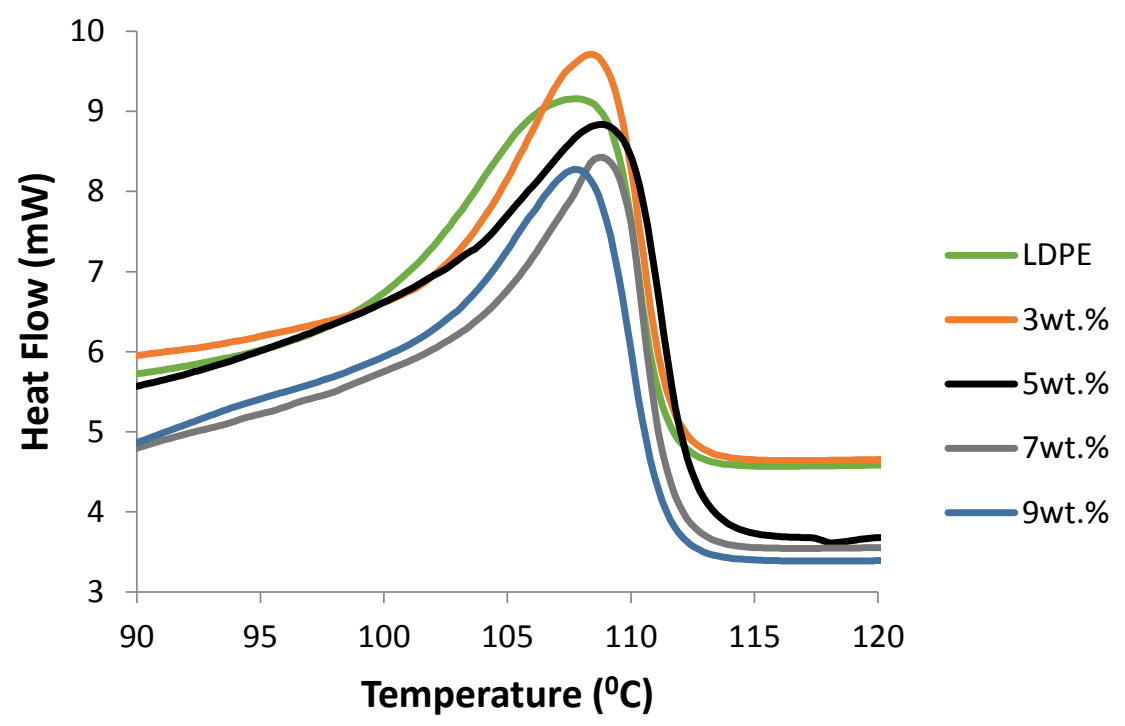

Figure 3: DSC results for LDPE and samples of LDPE containing decabromide diphenyl ether

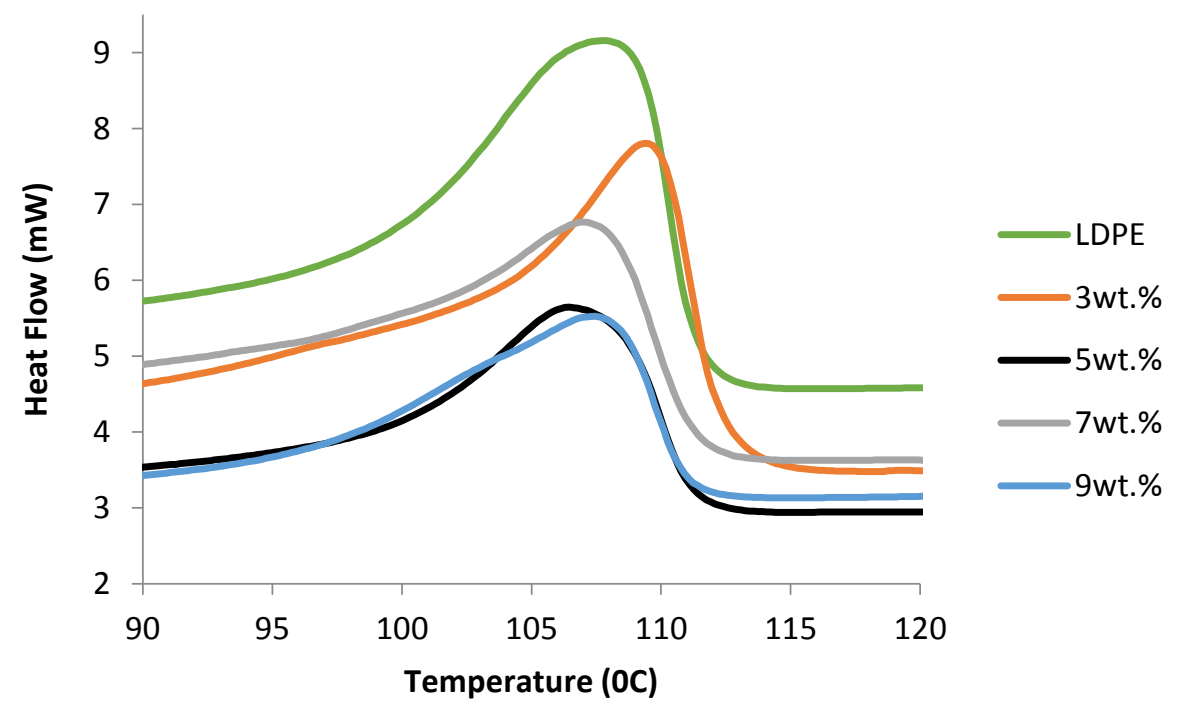

Figure 4: DSC results for LDPE and samples of LDPE containing magnesium hydroxide

The effects of both flame-retardants on the melting point and degree of crystallization of LDPE are shown in Tables 2 and 3. The percentage of crystalline regions of LDPE can be calculated from the equation 1: 


$$
x_{c}=\frac{\Delta H_{f}}{\Delta H_{f}^{0} X \varphi} X 100 \%
$$

Where $X_{C}$ is the percent of crystalline regions, $\Delta H_{f}$ is heat of fusion of the sample; $\Delta H_{f}^{\circ}$ is heat of fusion for $100 \%$ crystalline polyethylene which is found to be $290 \mathrm{~J} / \mathrm{g}$ [14] and $\varphi$ is weight percent of polyethylene in the produced samples.

Table 2: Thermal properties LDPE containing decabromide diphenyl bromide

\begin{tabular}{|c|c|c|c|c|}
\hline $\begin{array}{c}\text { Flame retardant } \\
\text { content }(\text { wt. \%) }\end{array}$ & $\begin{array}{c}\text { Melting } \\
\text { Temperature }\left({ }^{0} \mathrm{C}\right)\end{array}$ & $\begin{array}{c}\text { Crystallization } \\
\text { Temperature }\left({ }^{0} \mathrm{C}\right)\end{array}$ & $\begin{array}{c}\text { Heat of fusion } \\
\left(\Delta \mathrm{H}_{\mathrm{f}}\right)(\mathrm{J} / \mathrm{g})\end{array}$ & $\%$ Crystalinity \\
\hline 0 & 107.8 & 90.8 & 74.6 & 25.7 \\
\hline 3 & 108.4 & 94.4 & 46.0 & 16.4 \\
\hline 5 & 108.9 & 94.2 & 54.3 & 19.7 \\
\hline 7 & 108.9 & 94.9 & 63.5 & 23.5 \\
\hline 9 & 107.9 & 94.7 & 56.3 & 21.3 \\
\hline
\end{tabular}

Table 3: Thermal properties LDPE containing magnesium hydroxide

\begin{tabular}{|c|c|c|c|c|}
\hline $\begin{array}{c}\text { Flame } \\
\text { retardant } \\
\text { content }(\text { wt.\%) }\end{array}$ & $\begin{array}{c}\text { Melting } \\
\text { Temperature }\left({ }^{0} \mathrm{C}\right)\end{array}$ & $\begin{array}{c}\text { Crystallization } \\
\text { Temperature }\left({ }^{0} \mathrm{C}\right)\end{array}$ & $\begin{array}{c}\text { Heat of fusion } \\
\left(\Delta \mathrm{H}_{\mathrm{f}}\right)(\mathrm{J} / \mathrm{g})\end{array}$ & $\%$ Crystalinity \\
\hline 0 & 107.8 & 90.8 & 74.6 & 25.7 \\
\hline 3 & 109.5 & 92.9 & 57.4 & 20.4 \\
\hline 5 & 106.5 & 93.3 & 38.8 & 14.1 \\
\hline 7 & 107.3 & 93.9 & 37.7 & 14.0 \\
\hline 9 & 107.5 & 91.3 & 30.6 & 11.6 \\
\hline
\end{tabular}

Tables 2 and 3 show the melting point of LDPE slightly affected by the addition of flame-retardants additives; it increased from 107.8 to $108.9^{\circ} \mathrm{C}$ when $5 \mathrm{wt} . \%$ and $7 \mathrm{wt} . \%$ decabromide diphenyl bromide was added. On the other hand, the melting point of LDPE was increased from 107.8 to $109.5^{\circ} \mathrm{C}$ when $3 \mathrm{wt} . \%$ magnesium hydroxide was added, then it was slightly decreased when higher magnesium hydroxide contents were used. This may be related to poor distribution of the additives within the polymer melt due to increase in the melt viscosity as the melt flow index increased (Figure 2). It is obvious that there is a small effect of both flame-retardants additives on melting temperature of LDPE which in its turn will not adversely affect the ease of processing of LDPE. Furthermore, the percentage of crystallinity was initially decreased when decabromide diphenyl ether was added then it started to increase to reach $23.5 \%$ at $7.0 \mathrm{wt} . \%$ while the percentage of crystallinity decreased with increasing the magnesium hydroxide content. The increase in the degree of crystallinity of LDPE may be related to the fact that the additives act as nucleating agents where the decrease in the degree of crystallinity of LDPE may be attributed to the poor mixing process. It can be seen from the Table 2 and 3 that the crystallization temperature of LDPE was increased from 90.8 to $94.9^{\circ} \mathrm{C}$ and $93.9^{\circ} \mathrm{C}$ when $7.0 \mathrm{wt} . \%$ decabromide diphenyl bromide and $7.0 \mathrm{wt} . \%$ magnesium hydroxide were used, respectively. The increase in the crystallization temperature of LDPE is related to the fact that the flame retardants act as nucleating agents.

\subsection{Tensile properties of LDPE containing flame retardants}

In general, the tensile properties of LDPE are affected by the addition of flame-retardants, but this effect depends on the flame-retardant types and its content. The effects of flame-retardants and their content of the ultimate tensile strength, modulus of elasticity and ductility of LDPE are shown in Figures 5-7. 


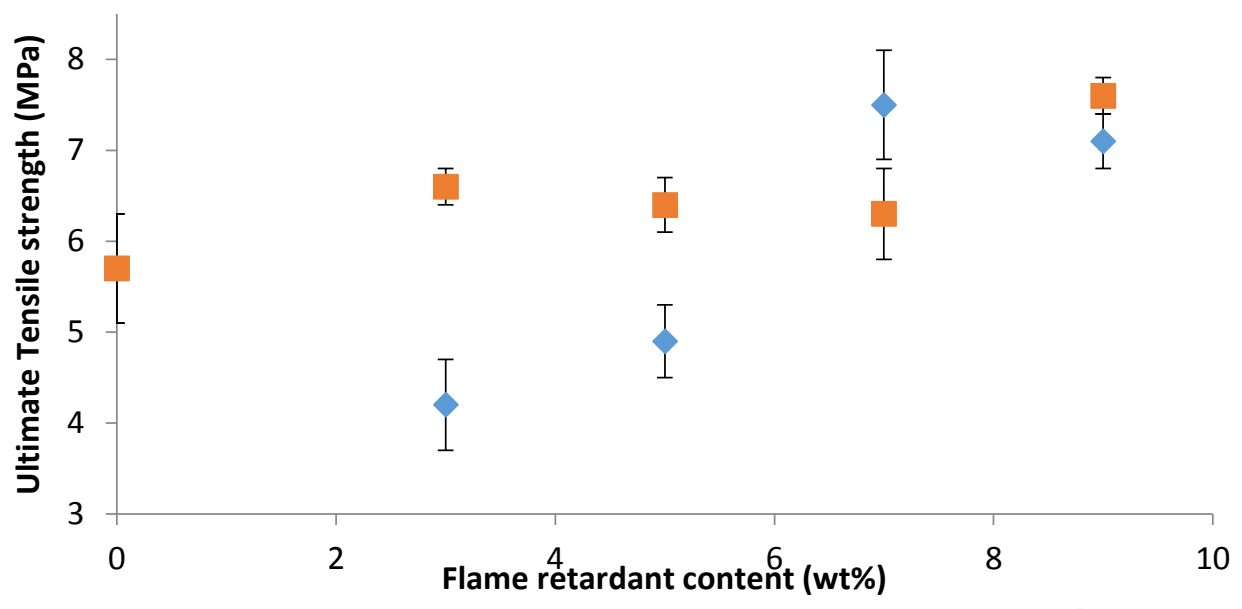

Figure 5: The Ultimate tensile strength of LDPE-flame retardants samples, where ${ }^{\star}$ and represent magnesium hydroxide-containing samples and decabromide diphenyl ether samples, respectively.

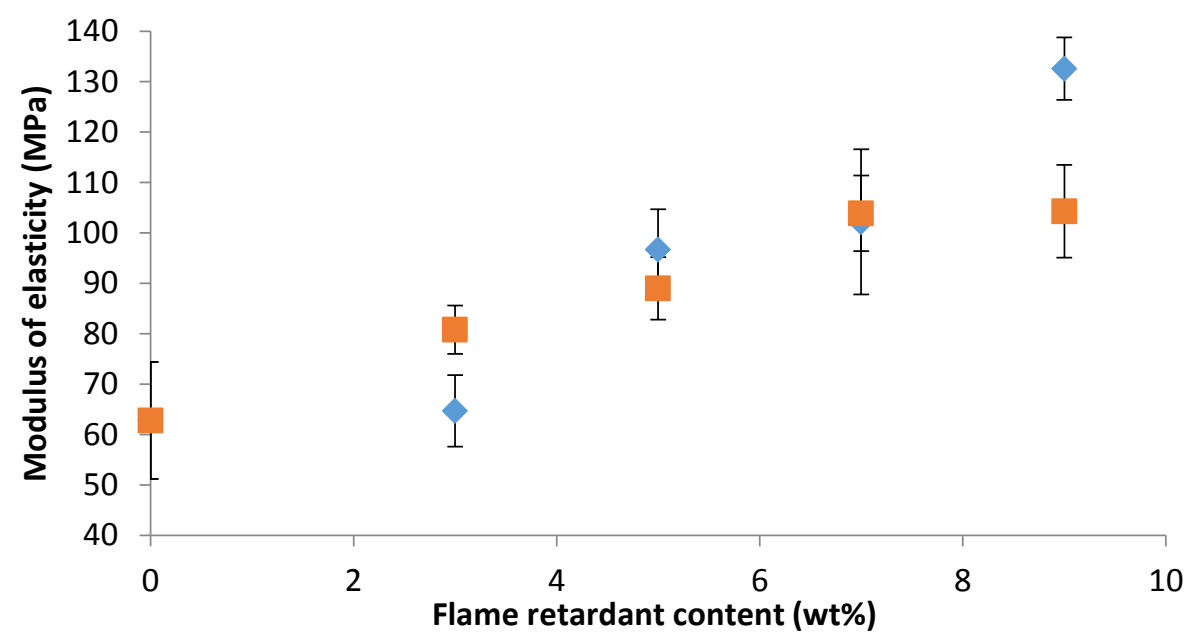

Figure 6: Modulus of elasticity LDPE-flame retardants samples, where and $\boldsymbol{\square}$ represent magnesium hydroxide-containing samples and decabromide diphenyl ether samples, respectively.

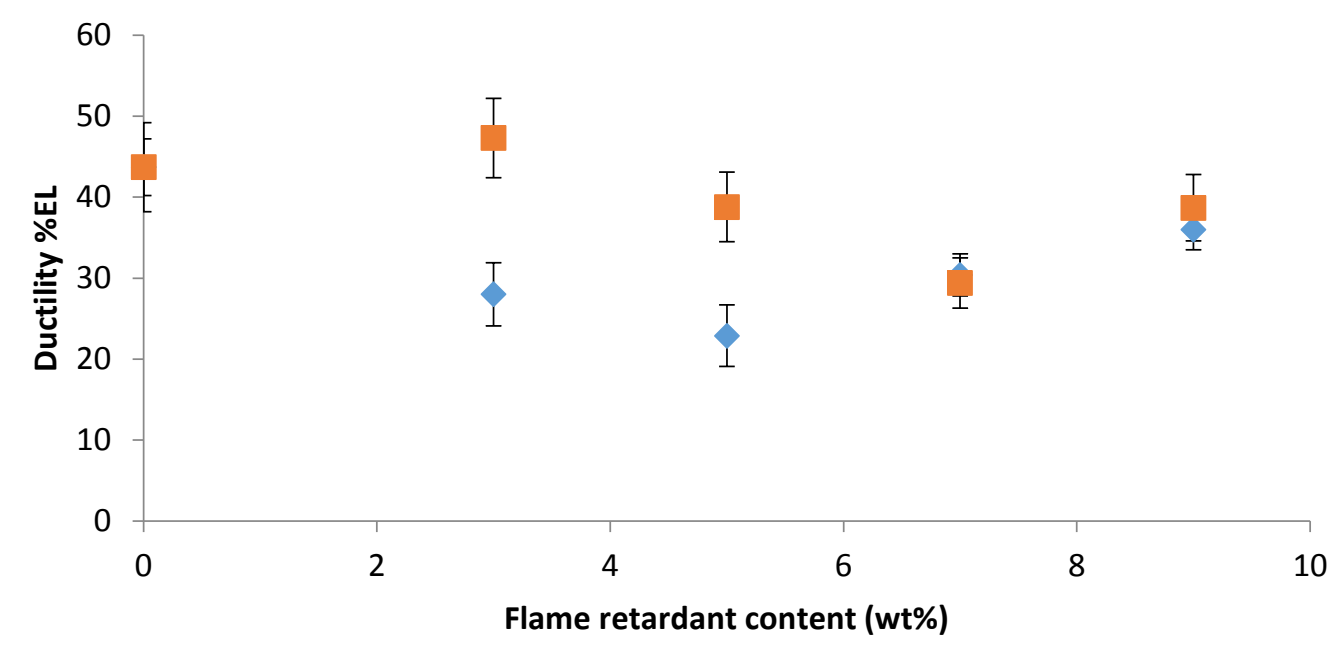

Figure 7: Ductility (\%EL) of LDPE-flame retardants samples, where and $\square$ represent magnesium hydroxide-containing samples and decabromide diphenyl ether samples, respectively. 
It can be seen from Figure 5 that the ultimate tensile strength of LDPE increases with the increase of decabromide diphenyl ether content to reach its maximum value at $9.0 \mathrm{wt} . \%$, but the addition of magnesium hydroxide results in a reduction of ultimate tensile strength then it starts to increase to reach its maximum value at $7.0 \mathrm{wt} . \%$. The modulus of elasticity of LDPE increases when both flame retardants are added as shown in Figure 6. The increase in the ultimate tensile strength and modulus of elasticity is related to the fact that the flame retardants act as load carries and they affect the degree of crystallinity of LDPE. It can be observed from Figures 5 and 6 that the samples containing decabromide diphenyl ether have higher ultimate tensile strength and modulus of elasticity than those containing magnesium hydroxide. This is related to the fact that the samples containing decabromide diphenyl ether may have better interfacial adhesion between the polymeric matrix and the additive compared with magnesium hydroxide additive. The reduction in tensile strength and modulus of elasticity of samples containing LDPE and aluminum hydroxide was observed, and the use of a silane coupling agent to improve the tensile properties of LDPE and aluminum hydroxide was reported [15]. The improvement in the ultimate tensile strength and modulus of elasticity of LDPE may be also related to the higher degree of crystallinity of LDPE, as shown in Tables 2 and 3, when decabromide diphenyl ether was used. The degree of crystallinity reduces the ability of movement of the molecular chains which increases the ultimate tensile strength and modulus of elasticity the produced samples.

On the other hand, the ductility of the produced samples was estimated in order to observe the effect of flame-retardants and their contents on the ductility of LDPE; this effect is clear in Figure 7. It is obvious that the ductility of LDPE decreases with the addition of flame-retardants up to $5.0 \mathrm{wt} . \%$ and $7.0 \mathrm{wt} . \%$ when magnesium hydroxide and decabromide diphenyl ether were used, respectively. The decrease in ductility of LDPE and the increase in modulus of elasticity of LDPE with increasing the flameretardants content is a normal relation between both properties, the increase in modulus of elasticity results in decrease of ductility of the polymeric matrix which could be attributed to the formation of dense structures within the polymeric matrix as the flame-retardants concentration increases, while the increase in the ductility of LDPE when higher flame retardants content were added may be related to poor mixing of flame retardants with LDPE.

\section{CONCLUSIONS}

The burning time of LDPE was found to decrease from 42.4 to 15.8 and 3.9 seconds when magnesium hydroxide and decabromide diphenyl ether were added, respectively. This was related to the formation of water vapor due to the decomposition of magnesium hydroxide and to the elimination of free radicals by halogen. The ultimate tensile strength and the modulus of elasticity of LDPE were increased to a significant amount due the role of additives which act as load carries, and due to the effect of the additives on the degree of crystallinity of LDPE.

\section{ACKNOWLEDGMENT}

Authors would like to thank Omega Plast for electrical and communication insulating pipes company for their support they have offered to this research.

\section{REFERENCES}

1. YiWang, D., Liu, Y., Wang, Y. U., Artiles, C. P., Hull, T. R., Proce, D. "Fire Retardancy of Reactively Extruded Intumescent Retardant Polyethylene System Enhanced by Metal Chelated". Polymer Degradation and Stability, 92, 1592-1598, 2007.

2. Vargas, E. R., Valdes, S. S., Tabla, O. P., Gutierrez, S. C., Nonell, J. M., Romas-devalle, L. F., Leon, A. L., Acosta, R. L. "Structural Characterization of LDPE/EVA Blends Containing Nanoclay-Flame Retardant Combinations". Journal of Applied Polymer Science, 123, 1125-1136, 2012.

3. Ahmad Ramazani, S. A., Rahimi, A., Frounchi, M., Radman, S. "Investigation of Flame Retardancy and Physical-Mechanical Properties of Zinc Borate and Aluminum Hydroxide Propylene Composites". Materials \& Design, 29, 1051-1056, 2008. 
4. Zanetti, M., Kashiwang, T., Falqui, L., Camino, G. "Cone Calorimeter Combustion and Gasification Studies of Polymer Layered Silicate Nanocomposites". Chemistry of Materials, 14, 881-887, 2002.

5. Huang, N. H., Chen, Z. J., Wang, J. Q., Wei, P. "Synergistic Effects of Sepiolite on Intumescent Flame Retardant Polypropylene”. eXPESS Polymer Letters, 4, 743-752, 2010.

6. Chen, X. L., Jiao, C. M., Wang, Y. "Synergistic Effects of Iron Powder on Intumescent Flame Retardant Polypropylene System”. eXPESS Polymer Letters, 3, 359-365, 2009.

7. Chen, L. and Wang, Y. "A review on Flame Retardants Technology in China. Part I: Development of Flame Retardants". Polymers for Advanced Technologies, 21, 1-26, 2011.

8. Costa, R. A., Wagnenknecht, U., Heinrich, G. "LDPE/Mg-Al Double Hydroxide Nanocomposites, Thermal and flammability properties". Polymer Degradation and Stability, 92, 1813-1823, 2007.

9. Arize, A. Eboatu, A. N., Udeozo, P. I., Ofora, P. U., Okoye, N. H., Amadi, E. G., Mougbo, M. O. "Improving the Flame Retardancy of Polypropylene and High-Density Polyethylene Using Aluminum Hydroxide". European Journal of Applied Sciences, 7, 192-194, 2015.

10.Li, Y. L., Kuan, C. K., Hsu, S. W., Chen, C. H., Kuan, H. C., Lee, F. M., Yip, M. C., Chiang, C. L. "Preparation, Thermal Stability and Flame-Retardant Properties of Halogen-Free Polypropylene Composites". High-Performance Polymers, 24, 478-487, 2012.

11. Beltrán-Ramírez, F.I., Ramos-deValle, L.F., Ramírez-Vargas, E., .Sánchez-Valdes, S., EspinozaMartínez, A.B., Martínez-Colunga, J.G., Rodríguez-Fernandez, O.S., Cabrera-Alvarez, E.N. and López-Quintanill, M.L. "Effect of Nanometric Metallic Hydroxides on the Flame Retardant Properties of HDPE Composites", Hindawi Publishing Corporation Journal of Nanomaterials, 2014, Available at: http://dx.doi.org/10.1155/2014/969184.

12.Zahang, Sh., Horrocks, A. R. "A Review of Flame Retardant Polypropylene Fibres". Progress In Polymer Science, 28, 1517-1538, 2003.

13. Guo, Y., Guo J., Huang Z. and Teng L., "LDPE/LLDPE/APP Intumescent Flame Retardant Systems: Molding Parameters and Properties". Advanced Materials Research, Vols. 317-319, $112-$ $115,2011$.

14.Lame, O., Vigier, G., Humbert S. "Polyethylene Yielding Behaviour: What is Behind the Correlation Between Yield Stress and Crystallinity?". Polymer, 50, 3755-3761, 2009.

15.Wang N., Xiang, D., Mo, P. and Lu, Y. "Flame Retardant Low-Density Polyethylene with Aluminum Hydroxide/Commercial Fire Retardants FR01 Synergistic System". Advanced Materials Research, Vols. 652-654, 485-489, 2013. 